\title{
Thermally populated intrinsic localized modes in pure alkali halide crystals
}

\author{
A. J. Sievers, ${ }^{1}$ M. Sato, ${ }^{2}$ J. B. Page, ${ }^{3}$ and T. Rössler ${ }^{4}$ \\ ${ }^{1}$ Laboratory of Atomic and Solid State Physics, \\ Cornell University Ithaca, NY 14853-2501 \\ ${ }^{2}$ Graduate School of Natural Science and Technology, \\ Kanasawa University, Kanasawa, Ishikawa 920-1192, Japan \\ ${ }^{3}$ Department of Physics, Arizona State University, Tempe, AZ 85287-1504 \\ ${ }^{4}$ Yingli Green Energy Europe GMBH, \\ Heimeranstr. 37, D 80339 Munich, Germany
}

(Dated: September 19, 2018)

\begin{abstract}
The possibility of thermal excitation of intrinsic localized modes (ILMs) arising from anharmonicity in ionic perfect crystals is studied numerically for realistic model systems in one and three dimensions. Implications are discussed for an interesting high-temperature feature seen in earlier inelastic neutron scattering experiments on single crystal NaI. The general conclusion is that ILM formation energies are far too large for thermal excitation of ILMs to account for the observed feature in a pure crystal.
\end{abstract}

PACS numbers: 63.20.Ry, 63.20.Pw, 65.40.-b, 63.20.dd 


\section{INTRODUCTION}

The discovery that some localized excitations in nonlinear perfect lattices can be stabilized by lattice discreteness has led to extensive studies of their characteristic features. $\underline{1}^{-\underline{7}}$ Experimental studies have focused on intrinsic localized modes (ILMs) driven in the steady state to overcome energy loss to the lattice. Since spin lattices are intrinsically nonlinear, the CW driving of antiferromagnetic instabilities has made it possible to create and destroy 1D intrinsic localized spin wave modes in the layered antiferromagnet $\left(\mathrm{C}_{2} \mathrm{H}_{5} \mathrm{NH}_{3}\right)_{2} \mathrm{CuCl}_{4}$. $\underline{\underline{8}} \underline{\underline{9}}$ Additional details on the creation and destruction of ILMs have been studied experimentally for macroscopic 1D E\&M transmission lines $\frac{10,11}{11}$ and micromechanical arrays. 12,13 The common thread between the microscopic and macroscopic systems is that after a driven instability generates many ILMs, a few become stabilized by locking to the driver frequency. Hysteresis and switching of ILMs have been demonstrated for both kinds of nonlinear lattices.

In a 2005 numerical study, Eleftheriou and Flach 14 addressed the problem of whether or not ILMs might be observable in thermal equilibrium. They used a highly simplified monatomic 2D model square lattice having a single scalar degree of freedom at each site. The interactions comprised nearest-neighbor quadratic and on-site quartic terms. For a fixed temperature the system was thermalized, and molecular dynamics (MD) calculations were used to generate time-dependent ensemble-averaged amplitude autocorrelation functions which were Fourier transformed to produce power spectra. Clear signatures of ILMs proved elusive, but by cooling the edge of their 2D lattice (via dissipation terms) so as to remove

running lattice modes, the authors were able to see the remaining stationary ILMs. They noted that it would be difficult to carry out a corresponding procedure in any realistic laboratory experiment.

Thus it was somewhat surprising that in 2009, inelastic neutron scattering measurements on NaI: 0.3 mole \% T $\ell$ I showed that at a temperature of about $570 \mathrm{~K}$ a localized vibrational excitation rapidly appears near the center of the large phonon gap between the transverse optic and acoustic branches, polarized along the [111] direction. ${ }^{15}$ This observation was interpreted as the appearance of a thermally generated ILM. Subsequent measurements at still higher crystal temperatures showed even more complex dynamical behavior, interpreted as a coherent rearrangement of ILMs in the atomic lattice. ${ }^{16}$

A recent series of numerical studies by Dmitriev and coworkers have considered the 
theoretical question of high temperature excitation of localized vibrations in alkali halide crystals. $\frac{17}{}$ Employing some of the nonzero temperature MD methods of Ref. 14 but using realistic interaction potentials, they argue via simulations on a $2 \mathrm{D}$ nonlinear lattice model that the vibrational lifetime of the light atoms in a system with a large harmonic phonon gap grows with increasing temperature, and they interpret this as evidence of ILMs. Their calculations did not use the edge-cooling technique of Ref. 14 to isolate the thermally generated stationary ILMs.

Most recently, inelastic neutron scattering experiments on the vibrational spectrum of $\mathrm{NaI}$ up to $700 \mathrm{~K}$ have been reported with no evidence for a high temperature gap mode $\underline{18}^{18}$ Given the variety of theoretical and experimental results, there is value in reassessing the likelihood of an experimentally significant fraction of thermally populated ILMs in alkali halide crystals.

Because of the simplicity of the ionic bond in alkali halides, they were one of the first crystal types whose interatomic potentials and vibrational dynamics were well studied. $\underline{19}^{-}$ The result is that the Born-Mayer-Coulomb shell model potentials yield accurate fits to measured phonon dispersion curves. ${ }^{20}$ Since the resulting ionic potentials are quite harmonic, ILM production in these systems requires large vibrational amplitudes in order for the nonlinearity to produce dynamical localization. In this report we examine the formation energy necessary to produce an ILM in the phonon gap of alkali halide crystals, a topic essentially unexplored in the literature. We find that the necessary formation energy is much too large to produce a measurable concentration of ILMs below the pure ionic crystal melting point.

\section{FORMATION ENERGY ESTIMATES FOR ILMS IN IONIC CRYSTALS}

We have computed the formation energy for two ionic crystal models, a 1D model for KI and a previously published 3D model ${ }^{21}$ for NaI. The large mass difference between the

positive and negative ions in each of these lattices produces a large frequency gap between the optic and acoustic harmonic phonon bands. 


\section{A. KI}

Early theoretical work on ILMs focused on 1D models and is reviewed in Ref. 22. As discussed there, the use of realistic interatomic full pair potentials $V(r)$ for ionic crystals, as opposed to harmonic and anharmonic spring models, rules out the existence of ILMs with frequencies above the maximum harmonic lattice frequency. This is because of the rapid softening of the interatomic forces with separation $r$. ILMs can readily form, however, in the phonon gaps of diatomic lattices.

Here we report calculated formation energies for gap ILMs in KI using interatomic BornMayer plus Coulomb (BMC) potentials. Specifically, the model is a 1D lattice of alternating masses $m$ and $M$ moving longitudinally, with nearest neighbors interacting via

$$
V_{m M}(r)=V_{M m}(r)=\lambda e^{-r / \rho}-\frac{q^{2}}{r}
$$

while second neighbors interact via pure Coulomb potentials

$$
V_{m m}(r)=V_{M M}(r)=\frac{q^{2}}{r} .
$$

More distant neighbors are assumed to be noninteracting. Periodic boundary conditions (PBCs) are used, for a lattice of $N=40$ particles.

Our parameter values were determined by fitting measured harmonic phonon dispersion curves $^{23}$ along the [111] direction for KI. The fit is excellent. Our model parameters are $m=39.1 \mathrm{amu}, M=127 \mathrm{amu}, \lambda=2.57 \times 10^{4} \mathrm{eV}, \rho=0.289 \stackrel{\circ}{A}$, and $q=0.90$ e. Minimization of the total potential energy yields the static lattice nearest-neighbor separation $a=3.50$ $\stackrel{\circ}{A}$. The lowest frequency of the harmonic optic phonon band is at the zone boundary, with $\omega_{z b m}=2.47 \times 10^{13} \mathrm{rad} / \mathrm{sec}$, equivalent to $16.3 \mathrm{meV}$ in energy units.

Using the rotating wave approximation (RWA) for the particles' time dependence,,$\frac{1,2,4,22,24}{1}$ we obtained accurate predictions for stationary ILM solutions of the classical equations of motion. Briefly, one assumes that the position of atom $n$ is described by

$$
r_{n}(t)=b_{n}+c_{n} \cos (\omega t)+r_{n}^{0}
$$

where $r_{n}^{0}$ is the equilibrium position. Substitution into the equations of motion, multiplication by either unity or $\cos (\omega t)$, followed by an integration over a single period yields a system of $2 N$ coupled nonlinear time-independent equations for the static and dynamic displacements $\left\{b_{n}\right\}$ and $\left\{c_{n}\right\}$. The equations are solved numerically, and the predictions are 
checked by MD simulations. It is straightforward to add higher harmonic terms to Eq. (3), but their contributions are typically down by an order of magnitude.

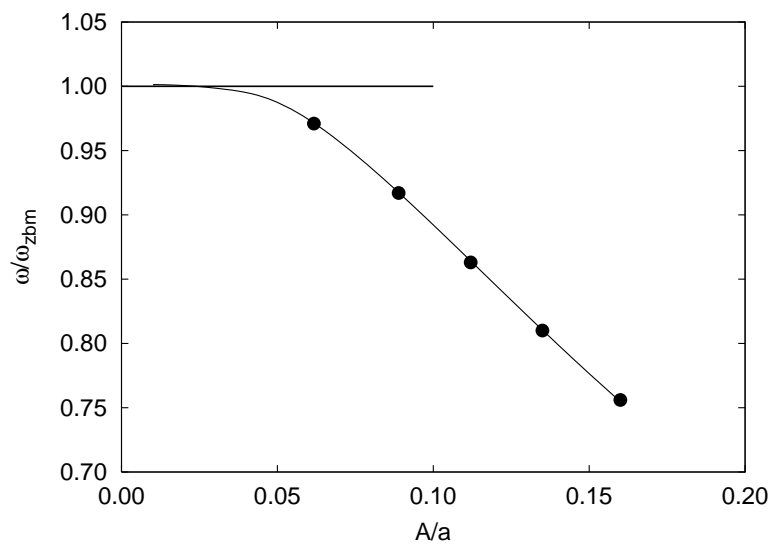

FIG. 1. Frequency vs. amplitude for gap ILMs in the 1D model for KI. The amplitudes $A$ are the dynamic displacements of the ILM's central particle and are normalized to the nearest-neighbor distance $a$. The frequencies are normalized to the harmonic phonon optic band minimum $\omega_{z b m}$. In this normalization, the harmonic phonon gap extends from 1 . to 0.51 . The circles denote the five ILMs for which formation energies are computed.

Figure 1 shows the predicted ILM frequency as a function of the ILM's central particle dynamic displacement $A$, along with symbols marking the ILMs whose formation energy were computed. The dynamic and static displacement patterns for the most localized of these are shown in Fig. 2, This ILM is represented by the rightmost circle on the curve of Fig. 1, and as one moves to the left along the curve, the ILM spatially broadens, eventually becoming the zone boundary mode. We did not run formal stability calculations (i.e., Floquet) for the ILMs, but MD runs showed that our highest frequency ILM $\left(\omega / \omega_{z b m}=0.97\right)$ persists for more than 900 periods, while that at $\left(\omega / \omega_{z b m}=0.92\right)$ is numerically stable for more than 200 periods. Our most localized ILM (at $\left.\omega / \omega_{z b m}=0.76\right)$ is numerically stable for $\approx 50$ periods, after which the central particle displacement slowly spreads into the lattice. The MD frequencies for the five ILMs in KI are within two to five percent of their RWA predicted values as the frequencies descend into the gap.

The ILM classical energy $E_{0}$ at $T=0 \mathrm{~K}$ is conveniently computed via two single timestep MD runs. First, we sum the predicted dynamic and static displacements at each site to obtain the ILM's initial displacement pattern $\left\{r_{n}(0)=b_{n}+c_{n}+r_{n}^{0}\right\}$, for which the kinetic 


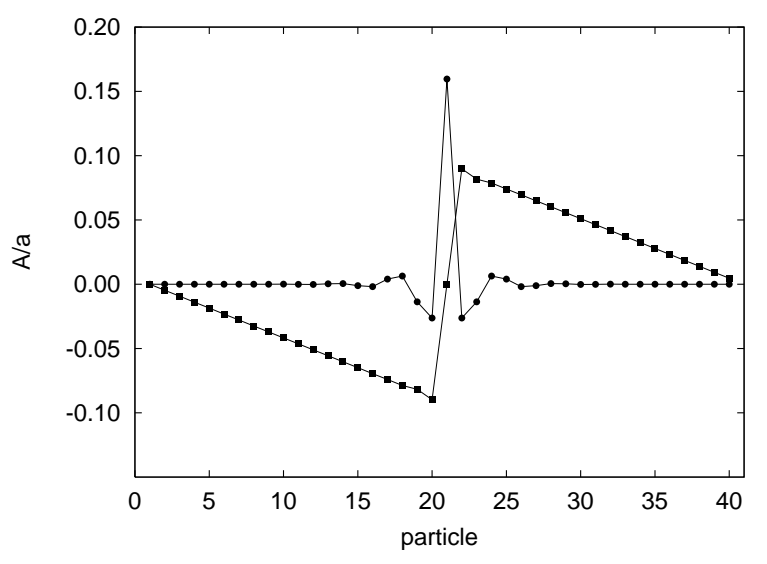

FIG. 2. Displacement patterns for the ILM at $\left(\omega / \omega_{z b m}, A / a\right)=(0.76,0.16)$ in the 40 particle 1D model for KI. The dynamic and static displacements are denoted by circles and squares, respectively. The actual displacements are longitudinal, but are plotted vertically for clarity. The static displacements vanish at the ends, due to the use of periodic boundary conditions.

energy is zero. Since the potential energy (PE) and forces are computed at every time step in MD before the particles move, the first timestep provides the full PE (including that from the static distortion) for the $t=0$ ILM configuration. Subtracting from this the similarly obtained PE for the equilibrium configuration of the lattice $\left\{b_{n}=c_{n}=0\right\}$ then yields the ILM's full classical energy.

Applying the above procedure for the five ILMs indicated on the curve of Fig. 1, we obtained the results shown by the crosses in Fig. 3, This figure also includes results for the 3D model of NaI, which will be discussed later. Here we focus on the energies for KI, shown in the lower left part of the figure. The results given by the crosses were obtained using the single-step MD calculations described above, and one sees that the energies are large, ranging from 70 to $300 \mathrm{meV}$. The temperature scale on the right-hand side of the figure shows that the corresponding equivalent temperature $T=E_{0} / k_{B}$ of the lowest-energy ILM, for $\left(\omega / \omega_{z b m}, A / a\right)=(0.97,0.062)$, is $858 \mathrm{~K}$, near the KI melting point of $954 \mathrm{~K}$. The other four energies are well above that temperature.

In addition to the full $E_{0}$ results, Fig. 3 gives two sets of KI results which follow from simple approximations, based on the assumed RWA time dependence of Eq. (3). Within the RWA the maximum kinetic energy of an ILM is given by the familiar harmonic approximation 


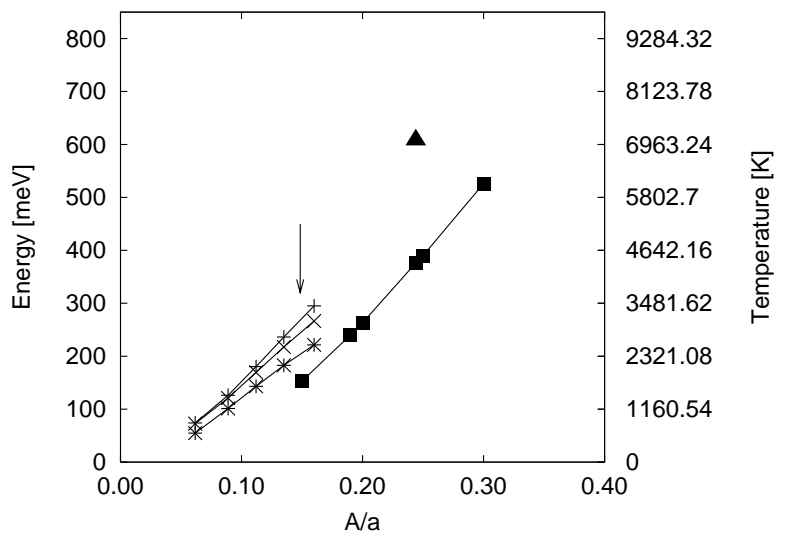

FIG. 3. ILM energies $E_{0}$ versus ILM amplitude, computed for the $1 \mathrm{D}$ KI model and the 3D NaI model. For KI, the crosses are for the full MD method described in Sec. IIA, and the $\times$ and star symbols are for the two approximation methods introduced there, namely $\mathrm{RWA}_{K E}$ and RWA $_{K E c e n t r a l}$, respectively. The squares give NaI results computed using the RWA KEcentral approximation, while the single triangle is the result of the better RWA $_{K E}$ approximation, with the sum in Eq. (4) going over the ILM central particle and two nearest neighbor shells. The energies $E_{0}$ are relative to the $T=0 K$ equilibrium configuration of the lattice. The vertical arrow is explained in the text.

result

$$
E_{R W A_{K E}}=\frac{\omega^{2}}{2} \Sigma_{n=1}^{N} m_{n} c_{n}^{2}
$$

where the sum is over the $N$ particles of the lattice. This approximation requires only the predicted frequencies and dynamic displacements. Despite the facts that an ILM has a static component $\left\{b_{n}\right\}$ which does not contribute to the above formula and also that the RWA is itself not exact, this approximation turns out to be quite good, as is seen by the $\times$ symbols in Fig. 3. As one expects, the results of this approximation become very close to the full $E_{0}$ results for our smallest-amplitude ILM at $A / a=0.062$. An even more approximate result is to include only the contribution from the ILM's central particle ( $A^{2}$ term) in the sum of

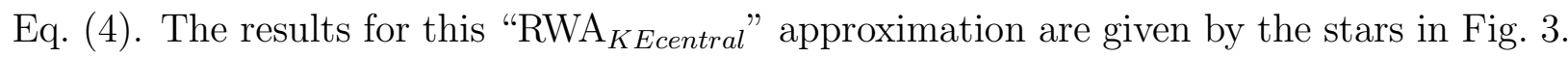

The more exact full energies $E_{0}$ and their two RWA approximation counterparts in Fig. 3 are seen to be quite close. More importantly, the results from all three methods cover nearly the same equivalent temperature range, much higher than the $570 \mathrm{~K}$ temperature at which the anomalous gap mode feature appears in the inelastic neutron scattering data of 
Ref. 15.

\section{B. $\mathrm{NaI}$}

To estimate the $T=0 \mathrm{~K}$ classical energy for an ILM in 3D NaI, we draw on the results presented for the 1D KI case treated above. Reference 21 summarizes the 3D model used for NaI, and additional details are given in Ref. 25. Briefly, a simulated annealing technique was combined with a rigid-ion two-body potential model of NaI to predict the properties of its ILMs for two different ionic lattice structures, namely zincblende and fcc. The predictions were verified with MD. The results are summarized in Fig. 4 of Ref. 21, which shows the ILM frequency versus amplitude curves for the two structures. As for the previous section, the amplitude is the predicted dynamic displacement of the ILM's central particle. The general behavior is similar for both lattice structures in that with increasing mode amplitude the ILM frequency drops farther into the harmonic phonon gap between the TO and TA branches. The difference between the curves for the two lattices is that for the zincblende structure the ILM frequency decreases much more rapidly with increasing ILM amplitude. For 3D NaI an amplitude threshold is observed at about $A / a=0.1$ (a similar, but smaller, threshold is seen for 1D KI in Fig. (1). For NaI it was found that at a relative amplitude of 0.244 the ILM normalized frequency is $\omega / \omega_{z b m}=0.95$, where $\omega_{z b m}$ is the frequency of the harmonic zone boundary TO mode in the [111] direction. For this ILM the predicted static and dynamic displacements are given for the central particle and four shells of neighbors in Table I of Ref. 21. This ILM had the longest lifetime, $\approx 200-250$ periods, via MD runs. For an ILM frequency of $\omega / \omega_{z b m}=0.90$, the lifetime decreased to $\approx 100$ periods. These numerical lifetimes are qualitatively similar to those given above for the 1D KI model over the same range of $\omega / \omega_{z b m}$. The NaI predicted and MD frequencies were plotted out to a relative amplitude $A / a$ of 0.3 in Ref. 21, but the ILM energies were not recorded.

The solid squares in Fig. 3] give energies for 3D NaI ILMs computed using the "RWA Ecentral" approximation defined below Eq. (4) and which we have seen works quite well for 1D KI. The ILM central particle amplitudes needed for this approximation were obtained from Fig. 4 of Ref. 21. Again following the results for KI, an improved estimate can be obtained from Eq. (4) by including more than just the ILM's central particle in the sum. The triangle in Fig. 3 gives the result for the ILM having relative amplitude $(A / a=0.244)$. The sum 
included the central particle and its two nearest shells of neighbors, with the displacements taken from Table I of Ref. 21. Similar to the 1D KI calculation, the complete full energy for this amplitude should be somewhat larger, and the energy curve should grow rapidly with increasing amplitude, just as for the central-particle estimate. One major difference between our energy results for 1D and 3D is highlighted by the arrow in Fig. 3, which shows the position of the 1D KI relative amplitude corresponding to the relative frequency $\omega / \omega_{z b m}=0.78$ of the experimentally measured NaI gap mode. Within the 3D ILM model for $\mathrm{NaI}$, this relative frequency of 0.78 shifts the arrow into a nonphysical region where the relative amplitude would be larger than $A / a=0.4$. This would correspond to a huge ILM energy (note that simply extrapolating the solid squares to $A / a=0.4$ would give a lower bound of $9000 \mathrm{~K}$ for the ILM's energy equivalent temperature) $\underline{\underline{26}}$

We have seen that the classical energy $E_{0}$ needed to create a mid-gap ILM in our 40 particle 1D KI model is of order $300 \mathrm{meV}$. We emphasize that this energy is relative to that for the $T=0 \mathrm{~K}$ classical lattice equilibrium configuration. As pointed out earlier, with decreasing amplitude the ILM spatially broadens, eventually becoming the harmonic zone boundary mode. At a high temperature, viz. $570 \mathrm{~K}$, and with no ILM present, the harmonic zone boundary mode has energy $k_{B} T=49 \mathrm{meV}$. As a first approximation then, we imagine the ILM's "parent" zone boundary mode converting to the ILM, and thereby take the ILM formation energy to be $E_{F}=E_{0}-k_{B} T=295-49=246 \mathrm{meV}$. For the 3D NaI model the situation is different since the ILM's harmonic "parent" mode is the zbm at the L point along the [111] direction in the BZ. Since there are four symmetry-equivalent L points which can convert to the ILM, we take the formation energy in NaI to be $E_{0}-4 K_{B} T$. The ILM formation energy results are summarized in Table —.

In the configurational entropy model, the site-occupancy probability for a localized lattice excitation, be it a vacancy or ILM, is

$$
p(T)=\frac{1}{e^{T_{F} / T}+1}
$$

where $T_{F}$ is the formation temperature. Since our approximate ILM formation energy expressions above include small temperature corrections to $E_{0}$, we see that the corresponding formation temperatures are of the form $T_{F}(T)=E_{0} / k_{B}-c T$, where the constant $c=1$ or 4 for the 1D and 3D models, respectively. For NaI at $570 \mathrm{~K}$ and using the value of $E_{0}$ given in Table \, we obtain $T_{F}=4776 \mathrm{~K}$ and $p=2.3 \times 10^{-4}$. At the NaI melting point of $934 \mathrm{~K}$, 
TABLE I. ILM formation energies for the 1D KI model and the 3D NaI model, as listed in the first column. The second column gives the ILM frequency in units of $\omega_{z b m}$, the third column gives the corresponding dynamic amplitude of the ILM central particle in units of the nearest-neighbor distance $a$. The fourth column gives the calculated ILM energies $E_{0}$ plotted in Fig. 3, and column five gives our estimates of the ILM formation energies for $T=570 \mathrm{~K}$, using $E_{F}=E_{0}-k_{B} T$ for $\mathrm{KI}$ and $E_{F}=E_{0}-4 k_{B} T$ for $\mathrm{NaI}$, as discussed in the text.

\begin{tabular}{lcccc}
\hline \hline Model & $\omega / \omega_{z b m}$ & $A / a$ & $\begin{array}{c}E_{0} \\
(\mathrm{meV})\end{array}$ & $\begin{array}{c}E_{F}(570 K) \\
(\mathrm{meV})\end{array}$ \\
\hline KI 1D & 0.971 & 0.0617 & 74. & 25. \\
KI 1D & 0.917 & 0.0889 & 126. & 77. \\
KI 1D & 0.112 & 180. & 131. \\
KI 1D & 0.863 & 0.135 & 236. & 187. \\
KI 1D & 0.756 & 0.160 & 295. & 246. \\
NaI 3D & 0.950 & 0.244 & 608. & 412. \\
\hline \hline
\end{tabular}

these results become $T_{F}=3328 \mathrm{~K}$ and $p=2.8 \times 10^{-2}$.

\section{RELATIVE STRENGTH OF THE LOCAL MODE TO THE ZONE BOUND- ARY OPTIC MODE}

The idea here is to start from a 1D harmonic toy model with parameters chosen so that the dispersion curve roughly represents that for $\mathrm{NaI}$ in the [111] direction. The ILM is represented by a simple force constant harmonic defect mode centered on a Na atom whose two nearest-neighbor springs are weakened enough to produce a gap mode at 0.78 times the harmonic zone boundary frequency $\omega_{z b m}$, with the remaining springs unchanged. Denote the mode $\mathrm{Na}^{*}$. The properties of the dispersion curves, especially near the zone boundaries are then monitored numerically as the impurity concentration is increased. Our aim is to tie the effects on the mode spectrum directly to the number of gap modes without introducing the intermediate step of temperature. The final step is to track the strengths of the zone boundary mode and the gap mode when a random concentration of $\mathrm{Na}^{*}$ is present and compare the results with the experimental relative strengths shown in Fig. 2 of Ref. 15. 


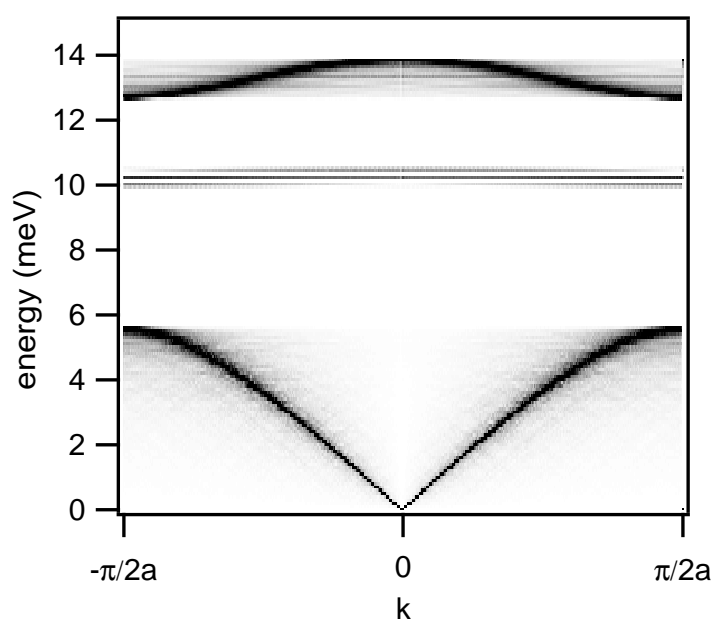

FIG. 4. Numerical dispersion curves for the 1000 particle 1D harmonic model NaI lattice with a $14 \%$ concentration of $\mathrm{Na}^{*}$ impurities.

To compare the strength of the harmonic gap mode to that of the zone boundary mode, we first obtained the normalized harmonic mode eigenvectors for the 1D model NaI host lattice, with 1000 particles. The eigenvectors were Fourier transformed to obtain $\omega(k)$. Next the force constant impurity was introduced, to reproduce the frequency of the assumed localized gap mode, and this was followed by a series of calculations of the normal modes for different random impurity concentrations. The harmonic eigenvectors for the defect lattice were Fourier transformed and their FT amplitudes were used to generate a perturbed "dispersion curve" as follows: for a mode of frequency $\omega$, the complex square amplitude of its FT at $k$ was added to a frequency histogram bin $(\omega, \omega+d \omega)$. The square root of sum of all such contributions was then plotted vs $k$, with the results shown in Fig. 4. One sees that the resulting curve is smeared out horizontally due to the fact that many of the modes are localized, so that their frequencies no longer correspond to a single $k$ point. This feature is most pronounced in the gap frequency region as expected, but it is also evident in the optic branch as the impurity concentration increases. Figure 4 is for $70 \mathrm{Na}^{*}$ particles $(14 \%$ concentration).

Panel (a) of Fig. [5] shows our resulting energy spectrum near the host lattice zone boundary as a function of $\mathrm{Na}^{*}$ impurity concentration. The figure covers the entire concentration range from zero to 500 impurities in our 1000 particle diatomic lattice, and two important features are evident: (i) the gap region is well defined over the whole impurity range and 


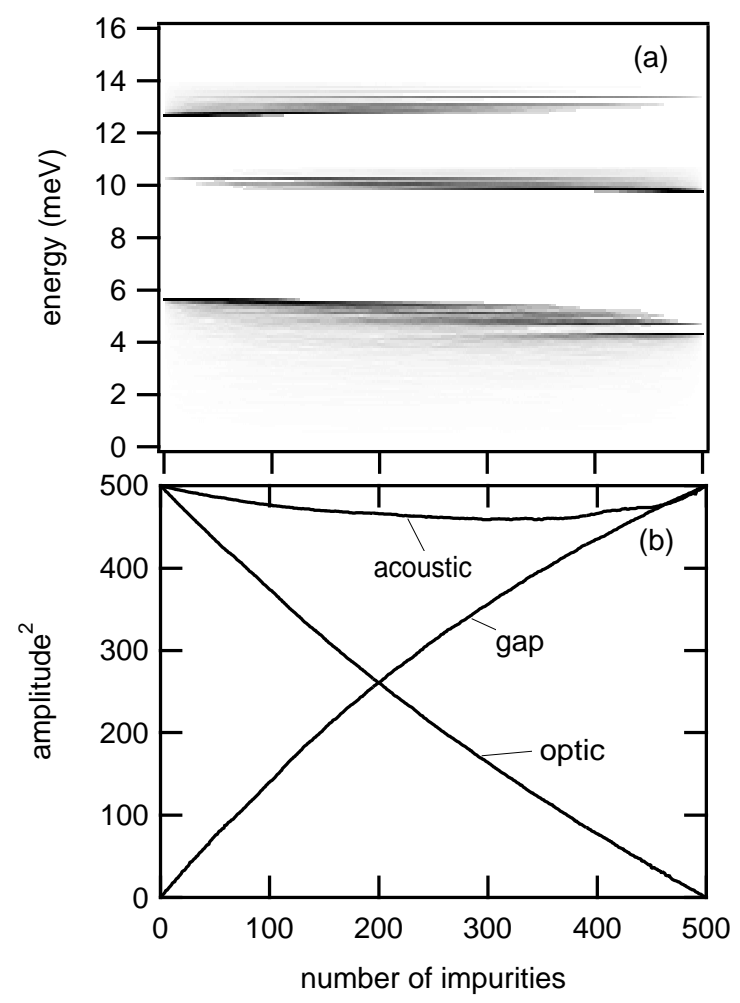

FIG. 5. (a) Energy dependences of optic and acoustic zone boundary modes and the gap mode for a force constant defect in the 1D harmonic model diatomic lattice vs the number of $\mathrm{Na}^{*}$ impurities. (b) Integrated strengths at the zone boundary in each of the three energy regions vs. impurity number.

(ii) the optic branch loses strength as the strength of the gap mode increases. We are particularly interested in the lower concentration range. To examine the energy dependence of our results more quantitatively, panel (b) of Fig. 5 plots the integrated strength in each of three energy regions versus concentration. Two key features are evident: (i) The zone boundary strength coming from the acoustic branch is fairly constant over the entire concentration range and (ii) the strengths of the gap band and zone boundary optic band are interconnected. Note that these two strengths are equal at an impurity number of 200, corresponding to about $40 \% \mathrm{Na}^{*}$. The gap mode strength is about half that of the optic mode when the number of impurities is 126 , corresponding to a concentration of about $25 \%$.

Because of the interconnection between the gap mode strength and the optic band strength at the zone boundary, these mode-counting results strongly suggest that the high temperature concentration of gap modes observed in the neutron scattering experiment is 


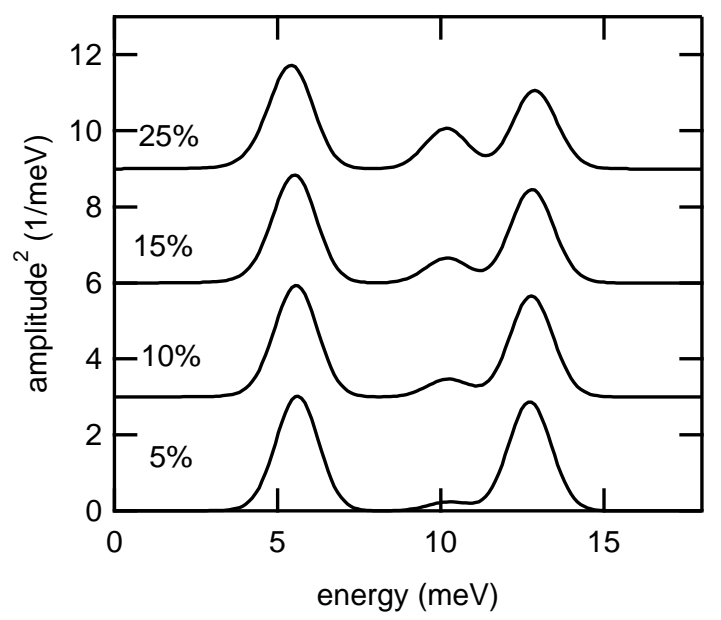

FIG. 6. Slices of the zone boundary data of panel (a) of Fig. 5 examined with a Gaussian resolution function with $1.5 \mathrm{meV}$ FWHM. The impurity concentrations are given in the figure, and the area under each trace is normalized to unity. The curves are shifted for clarity.

large, rather than small as was previously assumed. $\stackrel{15}{ }$ To double check this conclusion we took a Gaussian resolution function with $1.5 \mathrm{meV}$ FWHM and scanned it through the spectra shown in panel (a) of Fig. 5 for different concentrations. The results are given in Fig. 6. Note that the gap mode strength is $\approx 1 / 2$ of the optic zone boundary mode strength, as represented by $25 \%$ in Fig. 6. Clearly the impurity concentration producing the gap modes must be comparable with the concentration of normal $\mathrm{Na}$ ions producing the optic modes. In addition the low resolution representation of the zone boundary mode spectrum in Fig. 6] shows that the gap modes could be "seen" in either the 10 or $15 \%$ concentration spectra.

So far in this section, we have not invoked the concept of temperature. If we assume that the concentration of $\mathrm{Na}^{*}$ is due to a thermally activated process with activation energy $k_{B} T_{F}$, the configurational entropy model for vacancy production [Eq.(5)] would apply. What is the activation energy needed to produce a $25 \%$ concentration of impurities at about 570 K? From our present 1D zone boundary mode counting calculation, the gap mode strength at $25 \%$ impurities in the lattice is $1 / 3$ and that of the optic branch is $2 / 3$. Hence $25 \%$ corresponds to a probability of 0.33 , and according to Eq. (5) for $T=570 \mathrm{~K}$ we have $T_{F}=404 \mathrm{~K}$. Note that for this small energy barrier, the room temperature impurity concentration would be $\approx 20 \%$, but no such evidence of gap mode scattering is found at either 300 or $473 \mathrm{~K}$ in the neutron scattering data. 15 In the experiment, the gap modes "seem" to appear suddenly in 
that temperature range, with a $25 \%$ concentration.

\section{DISCUSSION AND CONCLUSIONS}

How does the observed high temperature gap mode feature in NaI compare with the work reported by others for $\mathrm{NaCl}$ type crystals? In the third paper of Ref. 17, Kistanov and Dmitriev conclude from MD calculations of the density of phonon states at elevated temperatures that ILMs (discrete breathers) do exist for crystals with the $\mathrm{NaCl}$ structure that have a gap between their optic and acoustic phonon branches. Our inspection of their Fig. 3(d), which they use to support their claim, indicates that what they have found at high temperatures is not due to ILMs but is instead the modulation of the optical density of states by the low-lying acoustic density of states due to the nonlinearity. We note that the widths of the sum and difference frequency sidebands, as measured from the peak in the optical density of states, each correspond to the width of the acoustic density of states in the same figure, as would be expected from this interpretation. There is no evidence of ILMs in this spectrum.

On the experimental side, the inelastic neutron scattering results for NaI between $300 \mathrm{~K}$ and $700 \mathrm{~K}$ at $100 \mathrm{~K}$ intervals, presented in Ref. 18, failed to show any evidence of a gap mode at $600 \mathrm{~K}$ at the zone boundary $\mathrm{L}$ point for energy spectra taken at $\mathbf{Q}=(2.5,2.5,1.5)$. (Measurements were not made at $555 \mathrm{~K}$.) The authors did observe the gap region between the optic and acoustic branches to fill in at high temperatures as would be expected from the modulation of the optical density of states by the acoustic modes, as mentioned in the preceding paragraph.

What we have described in this paper is consistent with these negative findings. For the 1D Born-Mayer-Coulomb model of KI, we computed the ILM energies $E_{0}$ by three methods. Two of them are simple approximations that require knowledge of only the ILM's frequency and dynamic displacements, which are straightforward to predict in the RWA. The third and most exact method is a direct calculation of the $t=0$ classical potential energy at the first step of an MD run that starts from the RWA-predicted full ILM displacement pattern, static plus dynamic. For ILMs in KI, the three methods were found to be in good agreement, with the more exact method giving an energy $E_{0}$ of about $300 \mathrm{meV}$ for an ILM whose frequency is well within the harmonic phonon gap $\left(\omega=0.76 \omega_{z b m}\right)$. Of the two approximate methods, 
the roughest requires the predicted frequency and dynamic displacement of only the central particle, and for the same ILM in KI it yields about $220 \mathrm{meV}$, about $25 \%$ below the more exact value.

For NaI, we drew on the previously published 3D rigid ion model ILM results of Ref. 21 . Using that paper's predicted ILM frequencies and central particle dynamic displacement in our roughest approximation method for an ILM near the top of the gap at $\omega / \omega_{z b m}=0.95$, we estimate the energy $E_{0}$ to be near $400 \mathrm{meV}$. Using the better of the two approximation methods raises this figure to just above $600 \mathrm{meV}$. But this ILM is near the top of the gap, and we argued in Sec. IIB that for an ILM deeper in the NaI phonon gap, the energy would be of the order of $1000 \mathrm{meV}$. This value is comparable to that measured for the formation of vacancies in $\mathrm{NaI}(1840 \mathrm{meV}) \stackrel{27}{\underline{27}}$ Such large energies predict that both ILM and vacancy concentrations would necessarily be very small, even near the NaI melting temperature of $934 \mathrm{~K}$. At the same time, our calculation in Sec. III of the relative strength of a hypothetical harmonic defect mode to that of the harmonic zone boundary mode in a 1D harmonic diatomic crystal would require a defect concentration of about $25 \%$ to match the experimental inelastic neutron scattering results at $570 \mathrm{~K}$. We conclude that thermally excited ILMs cannot be the source of the high temperature local mode observed in the phonon gap of NaI.

More generally, the MD simulations of Ref. 17, as described earlier, show that the modulation spectrum of the optic modes produced by the acoustic modes can be expected to have an undesirable experimental consequence. In a vibrational lattice with wide harmonic frequency gaps, this process will necessarily mask the experimental observation of the low concentration of ILMs lurking there. To expose ILMs experimentally in atomic lattices it appears necessary to lower the temperature and externally drive the lattice. $\underline{24}^{2} 28$

\section{ACKNOWLEDGMENTS}

A.J.S acknowledges the hospitality of the Department of Physics, Arizona State University, where much of this work was completed. He also thanks S. V. Dmitriev for helpful correspondence. A.J.S was supported by Grant NSF-DMR-0906491, and M.S. was supported 
by JSPS-Grant-in-Aid for Scientific Research No. 25400394.

1 A. S. Dolgov, Fizika Tverdogo Tela 281641 [Sov. Phys. Solid State 28, 907 (1986)].

2 A. Sievers and S. Takeno, Phys. Rev. Lett. 61, 970 (1988).

3 J. B. Page, Phys. Rev. B 41, 7835 (1990).

4 S. Flach and C. R. Willis, Phys. Repts. 295, 182 (1998).

5 R. Lai and A. J. Sievers, Phys. Repts. 314, 147 (1999).

6 D. K. Campbell, S. Flach, and Y. S. Kivshar, Physics Today 57, 43 (2004).

7 S. Flach and A. Gorbach, Phys. Repts. 467, 1 (2008).

8 M. Sato and A. J. Sievers, Nature (London) 432, 486 (2004).

9 J. P. Wrubel, M. Sato, and A. J. Sievers, Phys. Rev. Lett. 95, 264101 (2005).

10 L. Q. English, R. B. Thakur, and R. Stearrett, Phys. Rev. E 77, 066601 (2008).

11 L. Q. English, F. Palmero, A. J. Sievers, P. G. Kevrekidis, and D. H. Barnak, Phys. Rev. E 81, 046605 (2010).

12 M. Sato, B. E. Hubbard, and A. J. Sievers, Rev. Mod. Phys. 78, 137 (2006).

13 M. Sato, S. Imai, N. Fujita, S. Nishimura, Y. Takao, Y. Sada, B. E. Hubbard, B. Ilic, and A. J. Sievers, Phys. Rev. Lett. 107, 234101 (2011).

14 M. Eleftheriou and S. Flach, Physica D 202, 1422005.

15 M. E. Manley, A. J. Sievers, J. W. Lynn, S. A. Kiselev, N. I. Agladze, Y. Chen, A. Llobet, and A. Alatas, Phys. Rev. B 79, 134304 (2009).

16 M. E. Manley, D. L. Abernathy, N. I. Agladze, and A. J. Sievers, Nat. Sci. Repts 1, 10 (2011).

17 L. Z. Khadeeva and S. V. Dmitriev, Phys. Rev. B 81, 214306 (2010); 84, 144304 (2011); A. A. Kistanov and S. V. Dmitriev, Fizika Tverdogo Tela 54, 1545 (2012) [Phys. Solid State, 54, $1648(2012)]$.

18 M. Kempa, P. Ondrejkovic, P. Bourges, J. Ollivier, S. Rols, J. Kulda, S. Margueron, J. Hlinka, J. Phys.: Condens. Matter 25, 055403 (2013).

19 N. W. Ashcroft and N. D. Mermin, Solid State Physics (Saunders College, Philadelphia, 1976).

20 H. Bilz, D. Strauch, and R. K. Wehner, Vibrational Infrared and Raman Spectra of Non-Metals, Handbuch der Physik, Vol. XXV (Springer-Verlag, Berlin, 1984).

21 S. A. Kiselev and A. J. Sievers, Phys. Rev. B 55, 5755 (1977). 
22 A. J. Sievers and J. B. Page, in Dynamical Properties of Solids, edited by G. K. Horton and A. A. Maradudin (North Holland, Amsterdam, 1995), Vol. 7, p. 137.

23 See, for instance, H. Bilz and W. Kress, Phonon Dispersion Relations in Insulators (Springer, Berlin, 1979).

24 T. Rössler and J. B. Page, Phys. Rev. B 62, 11460 (2000).

25 S. A. Kiselev, A. J. Sievers, G. V. Chester, Physica D: Nonlinear Phenomena 123, 393 (1998).

26 We note that it is the higher point symmetry of the fcc lattice relative to the zincblende lattice which suppresses the anharmonic contribution to the vibration and forces such a large amplitude to produce an ILM near the middle of the gap.

27 K. Tharmalingam, J. Phys. C:Solid St. Phys. 3, 1856 (1970)

28 T. Rössler and J. B. Page, Phys. Lett. A 204, 418 (1995); Physica B 219-220, 387 (1996). 